\title{
Requirements for mobile photoware
}

\author{
Morgan Ames • Dean Eckles • Mor Naaman • \\ Mirjana Spasojevic $\cdot$ Nancy Van House
}

Received: 11 May 2008/Accepted: 9 March 2009/Published online: 6 June 2009

(c) The Author(s) 2009. This article is published with open access at Springerlink.com

\begin{abstract}
What is the future of digital imaging? Mobile imaging technologies have been changing rapidly and will continue to do so. We explore new developments in cameraphone photography with the goal of improving the design of the next generation of mobile imaging devices. We equipped 26 diverse participants with cameraphones, photo uploading and sharing software, and access to online photo-accounts for 3-5 months. This study allowed us to identify emerging practices in mobile photoware. We report on new and continuing practices across the lifespan of photos in this new imaging environment, including image capture, upload, annotation, archiving, sharing, and viewing. Based on these results, we develop design criteria and implications for designers and makers of mobile devices, mobile imaging and sharing software, and desktop and online photo software.
\end{abstract}

Keywords Photography · Cameraphones · Photoware · Photo sharing $\cdot$ Imaging $\cdot$ Mobile phones

\footnotetext{
M. Ames $(\bowtie) \cdot$ D. Eckles

Department of Communication, Stanford University, 450 Serra Mall, Stanford, CA 94305-2050, USA

e-mail: morganya@stanford.edu
}

M. Ames - D. Eckles $\cdot$ M. Spasojevic

Nokia Research Center, 955 Page Mill Rd, Suite 200,

Palo Alto, CA 94304, USA

\section{Naaman}

School of Communication and Information, Rutgers University,

4 Huntington St., New Brunswick, NJ 08901, USA

N. Van House

School of Information, University of California,

102 South Hall, Berkeley, CA 94720-4600, USA

\section{Introduction}

Personal photography is one of the most successful mobile technologies of the last century. Not only has digital photography has rapidly supplanted film photography, but ever-present cameraphones may be poised to replace standalone digital cameras in many of their roles. The ubiquitous cameraphone brings new opportunities to media capture, as these devices are always at hand, and increasingly programmable, network-connected, and context-aware. Rapid improvements in image quality may substantially increase cameraphones' potential. The recent appearance, in the US and elsewhere, of affordable data plans (i.e., Internet connectivity) for mobile phones and of popular Internetfocused devices such as the Apple iPhone further alleviate a major adoption barrier.

Cameraphone use has diverged in some ways from that of other digital cameras. This is partly due to the facts that cameraphones are always at hand, and that they enable instant communication of pictures. Another cause of divergence has been the historically low quality of cameras in phones. Now, however, as cameraphones have better image quality, the combination of cameraphones with online sharing and storage systems holds even further promise.

Previous studies [1-5] have investigated aspects of early cameraphone use. This study, conducted at a time when cameraphones are more widespread and higher-quality, aims to provide a more complete view of current and emerging mobile imaging practices, with an eye to making practical recommendations to guide the design of the next generation of mobile imaging applications and help their adoption.

Our larger concern is how mobile imaging is changing photographic activity, and the associated design needs; and 
what this study may suggest about how "ordinary" (as opposed to technology-centric) people are beginning to use mobile devices.

In this article, we use the data from our empirical study to reflect on emerging uses of ubiquitous photography and design implications. We borrow the term "photoware" from Frohlich et al. [6]. They used this term to refer to groupware for collaboration around photos. We use the term photoware comprehensively: by "mobile photoware," we mean technology to suit all phases of a photograph's life, including taking, uploading, organizing and annotating, sharing, viewing, and archiving, which can each involve consideration of communication and collaboration. This breadth presents unique challenges in research and design.

This paper presents the results of a 5-month study of cameraphone practices. We equipped 26 "ordinary" people with a holistic imaging solutions, including high-end Nokia cameraphones, photo-upload software (ZoneTag), a photobrowsing application (Zurfer), accounts on the photo sharing site Flickr, and unlimited data plans. We report on their image capture, upload, organization, sharing, and mobile viewing practices, and we discuss key factors that influenced adoption and use. We particularly note the design decisions in the hardware and the software that most affected use and highlight the resulting design requirements. Our contributions include an unusually long-term study with diverse set of ordinary users, rather than early adopters of the technology over a short time, as tends to be the case with typical studies.

\section{Related work}

The study of personal photography and new photographic practices has spanned many years, technologies, and disciplines. Previous studies in visual sociology have reported on family photography with film cameras [7-9]. In humancomputer interaction, a number studies have examined practices around personal photographs. Rodden and Wood [10] studied a desktop photo organization system, focusing on organization and retrieval but also noting changes in capture behavior due to digital imaging, which was then still relatively new. Frohlich et al. [6] focused on viewing and sharing photos, studying remote and co-present sharing of both physical and digital images. Crabtree et al. [8] explored conversation styles around face-to-face photosharing with the goal of improving remote digital sharing. Kirk et al. [11] explore the "photowork" that users do with their digital images, including reviewing, editing, searching, and browsing. More recent research has addressed online photo-sharing $[4,5,12]$.
Some cameraphone-related research projects (e.g., [1317]) have developed innovative systems using the new capabilities of cameraphones. These studies often revolved around a specific implementation and did not investigate general photographic practices. However, several studies have explored general cameraphone use. Kindberg et al. [2] explored motivations for picture-taking and sharing with cameraphones along functional/affective and individual/social dimensions. Okabe and Ito [1] investigated mobile practices in Japan, and Koskinen et al. [3] explored mobile photo-sharing via MMS among teenagers in Finland.

Several studies examined specific aspects of online sharing of cameraphone images. Most related to our work here, Van House et al. [4, 18] studied kinds of images taken and patterns of sharing with cameraphones and the MMM upload software among a graduate student cohort at UC Berkeley, and among more general cameraphone and Flickr users. Ames and Naaman [19] examined motivations for tagging in mobile devices using ZoneTag and Flickr. Ahern et al. [13] examined users' privacy considerations around media captured with a mobile device and shared via Zonetag and Flickr.

Like Kirk et al., we take a holistic approach, studying a complete cycle of photographic activities aside from the capture itself. Like Van House et al. [4, 5], we introduced users to new cameraphone technologies, with some significant differences: cameraphones are now widespread and most of our users had at least some experience with cameraphones; some participants had already voluntarily adopted one of our technologies, Flickr; our users were not university students; and the state-of-the-art has changed dramatically since their study. We note the continuities with and divergences from these previous studies throughout our results.

\section{Method}

We borrow from, and extend, the methods used in previous studies on photography, especially Van House et al. $[4,5]$, focusing on open-ended interviews and data from a trial of 3-5 months. One of the most important aspects of this study is that our recruits were "ordinary" users (as opposed to technophiles, early adopters, or students) who were given access to the latest technology without cost or setup issues. The participants had used the technology long enough for the novelty to wear off and for them to find out how it would fit their lives and photo practices. Ours is a realistic study of the implications of the availability of high-quality cameraphones, with little restrictions on use. 


\subsection{Participants}

The initial participants were 26 adults chosen to represent a variety of user types, including parents, students or recent students, and professionals of various ages from midtwenties to mid-fifties. Fourteen participants were female. All participants had shared photos online in some fashion, and most were prior cameraphone owners (though their cameraphones were generally older and entry-level). Most participants had other digital cameras (their own or shared by family), and two participants had digital SLR cameras. All were already Cingular/AT\&T or T-Mobile subscribers, most without mobile Internet data plans.

These participants were members of five social groups, the members of each of which were loosely connected through the workplace or friendships; four of the groups had four or five members, and one (a group of parents) had eight members. We recruited social groups, rather than isolated individuals, because we were interested in whether participants would influence one another, as seen in other studies [4, 19]. We were also aware of earlier studies that had shown that people who were already interacting personally and professionally were inclined to share cameraphone images [4]. This method allowed us to involve participants with a range of experience with, and investment in, technology, from enthusiasts to late adopters. Another advantage of recruiting social groups was that we could hold mid-point focus groups of people who knew and were comfortable with one another, and who could talk about similarities and differences in their use within the study.

Much of the previous research on "snapshot" photography $[7,9]$ concentrated on family practices; photographic practices are strongly influenced by the presence of children in the household [4]. Most cameraphone studies [1-4] have looked at younger users. We wanted to explore cameraphone use also among parents and families, and thus specifically recruited parents for one of the five social groups. Overall, we recruited ten parents for our study.

Our pool of participants was balanced in terms of their experience with Flickr: 12 of the participants were prior users, some of them for a number of years. However, only two of the ten parents were prior Flickr users. Participants suggested that this was a function of age and computer use. Parents who weren't using computers at work didn't have time for Flickr-type applications at home. Also, several participants (one as young as 29) described Flickr as a "Facebook-type" application for a crowd younger than themselves.

Two of the participants dropped out of the study shortly after the beginning, and one participant dropped out after 1 month. Three others also dropped out along the way, leaving us with 20 participants at the end.

\subsection{Study chronology}

We interviewed participants when they joined the study, which was staggered between late March and May 2007, about their prior photographic practices of all types, including cameraphone use. We gave each participant a Nokia N80 cameraphone with photo sharing and data collection applications (described below) for the duration of the study. Participants were briefed on these applications and given a quick tutorial of ZoneTag and, if applicable, Flickr. We provided a Flickr "Pro" account, with unlimited upload and storage, to each participant for up to 6 months.

Participants used the Nokia N80 phone as their primary phone for at least 3 months. Eight participants accepted an option to extend the study for two more months. We chose to run the study for 3-5 months, longer than most other studies, in order to allow the novelty effect of the cameraphone to wear off and day-to-day use to set in.

Approximately halfway through the study, we conducted a series of focus groups, one for each social group with as many members of the group as we could gather. We asked about their photo use, answered questions about the hardware and software, and gave participants an additional piece of software, Zurfer, which facilitated viewing and commenting on Flickr photos from the mobile phone. We held five focus groups with a total of 17 participants.

At the end of the study, we conducted open-ended interviews with 12 participants (the remaining eight still in the study were unavailable during our timeframe), reviewing photographs taken during the study and discussing whether the participant's practices around photography had changed. We compensated all participants with gift checks equal to their data plan costs during the study.

When feasible, we used photo-elicitation [20] to ground the participants' responses in their actual photo activities by viewing and discussing their photos with them. Before the mid-point and final interviews, we reviewed their public Flickr images so that we could discuss them with participants. All interviews and focus groups were either video-recorded or (in a small number of cases) audiorecorded, and transcribed.

\subsection{Study artifacts}

\subsubsection{Nokia N80 cameraphone}

Each participant was provided with a Nokia N80 phone. The N80 has a three mega-pixel camera and can also capture VGA video. The phone has EDGE data connectivity as well as WLAN (802.11 "wi-fi"), but no GPS. The camera includes many settings normally found on mediumquality digital cameras, such as exposure compensation, 
white balance, and seven pre-defined shooting modes such as sports and night photography.

\subsubsection{Flickr}

Flickr [21] is a popular photo-sharing site owned by Yahoo! with over 8 million users. Flickr enables many forms of organization and navigation (sets/collections, tags, group pools, etc.). Users can specify a privacy policy for each photo such that the public or only designated "contacts," "friends," and/or "family" can access the photo. Visitors can leave comments on photos and about photographers that are then visible to all other viewers.

\subsubsection{ZoneTag}

ZoneTag [22] is a cameraphone application that allows users to upload photos from their phone to Flickr at the time of capture. ZoneTag encourages annotation on the phone, before upload, by suggesting tags as well as enabling the photographer to add titles and set the photo's privacy policy. ZoneTag automatically tags photos with the city or postal code in which the photos were taken, inferred from cell tower data when available (the geographic coverage of recognizable cell towers is dense at the area of the study but rather sparse elsewhere).

\subsubsection{Zurfer}

Zurfer [23] is a prototype cameraphone application that allows users to easily browse Flickr photos-including their own pictures and recent comments, their contacts' pictures, other pictures geo-coded as having been taken nearby, today's "interesting" pictures (a Flickr feature), and other custom channels - all on the phone. Participants received Zurfer during the focus group meeting. Only a few participants used Zurfer (but some of these heavily). We mostly report on use of Zurfer as a supplement to digital photography capture. When appropriate, we compare our findings to those in a more comprehensive study of Zurfer and its use in the wild [24].

\subsubsection{Monitoring software}

Each phone was equipped with an activity-logging tool that tracked the use and state of the phones. Collected information included the time and duration of voice calls, mobile messaging, and applications used (Web browser, camera, gallery, etc.). Unfortunately, we had near-complete logs for only 16 of our 20 (final) participants, as technical problems resulted in logs shorter than a week for the others. The quantitative data reported below is for these 16 participants.

\subsubsection{General phone use statistics}

All participants who completed the study used the Nokia N80 phone as their primary phone for the duration of the study. Some used the phone frequently, one averaging over 15 calls (made, received, and missed) a day, while a few averaged less than one call a day.

Six of the sixteen participants for whom we have quantitative data sent two or more text messages a day on average. The other ten sent fewer than two text messages a week on average. Interestingly, this was not correlated with calls made. Seven participants accessed the Web, on average, once a day or more (again, uncorrelated with calls or texting). Participants almost never used MMS except for one who averaged two MMS messages per day. Rates of photo-taking and sharing will be discussed in later sections.

\section{Mobile photoware}

In the following sections, we unpack the experience of our participants into themes and major factors that influenced technology adoption and use. Our primary findings are highlighted in bold. We divide activities involving mobile photoware into five (overlapping) categories tracing the life of a photograph: capturing, uploading, organizing and annotating, sharing, and mobile viewing. We also discuss technical considerations and adoption patterns. We conclude with a summary of the requirements for mobile photoware based on this analysis.

As noted above, in this study we took a holistic approach: we introduced participants to a set of hardware, software, and network services. In interviews, it was clear that most users, especially those who were not prior Flickr users, saw this assemblage of tools as one complete system. When we asked about ZoneTag, for example, they often answered about Flickr. The result is that, while we as researchers could often identify the causes of both satisfaction and dissatisfaction, users' interpretations and evaluations may not make these distinctions, as in rejecting a phone because its service network is slow. On the other hand, the "one system" approach was successful in that the combination often made each service more attractive than it was individually. For example, as we describe below, some who had not used Flickr previously liked it in conjunction with ZoneTag's uploading, but said they would not have used Flickr if they had to upload using other methods. More importantly, participants voiced desires that were not limited to a single system component, but more generally about what they wanted from the combination of hardware, software, and the network. 


\subsection{Image capture}

Here we discuss participants' capture practice and the influence of various aspects of the hardware/software assemblage. Those who actually used the cameraphone liked having it as an always-present camera. Some increased or changed their picture-taking.

\subsubsection{Image content}

As other cameraphone studies have found [1-5], a consequence of having a capture device available at all times is that more pictures of more kinds are taken in more settings, including some settings that are not frequently seen with other cameras. Figure 1 presents an array of such images. Several participants expressed delight at being able to take pictures of interesting sights or personally meaningful events throughout their day (Fig. 1a). Some took expressive (artistic or humorous) pictures with their cameraphones (Fig. 1c) [2, 4]. Photographs for documentation-a new haircut, a good meal, interesting graffiti-were especially prevalent (Fig. 1b). One participant, who had memory problems due to prior concussions, used his cameraphone to document events major and minor. The cameraphone helped him give order to his life by giving him a chronology of his experiences.
Participants liked that the cameraphone was always available, and often more acceptable than a "real" camera in settings where they would feel uncomfortable taking pictures with a camera, such as at work, in shops, in dressing rooms, at lectures, and on the street. As one participant reported, a presence of a professional looking camera tends to make others shy about picture taking: "People duck with the SLR." This convenience and acceptability were important for the increased frequency and variety of images.

\subsubsection{Convenience}

The factors that elicited the most enthusiasm, across the board, were the convenience of the ever-present cameraphone and the easy uploading with ZoneTag. The specific implementation of this functionality in this assemblage of hardware and software was, however, frustrating for many users. The camera was slow to start up and suffered from shutter lag, characteristics shared by many other current cameraphones. Participants described the camera as "sluggish" and "laggy," and were frustrated when they weren't able to capture a fleeting moment:

"Like 'That didn't come out' and they've walked away."
Fig. 1 Sample photos. a "structure" (art), b street art, c child, $\mathbf{d}$ new baby, e sign on plant, $\mathbf{f}$ friends, $\mathbf{g}$ MRI, $\mathbf{h}$ new tattoo
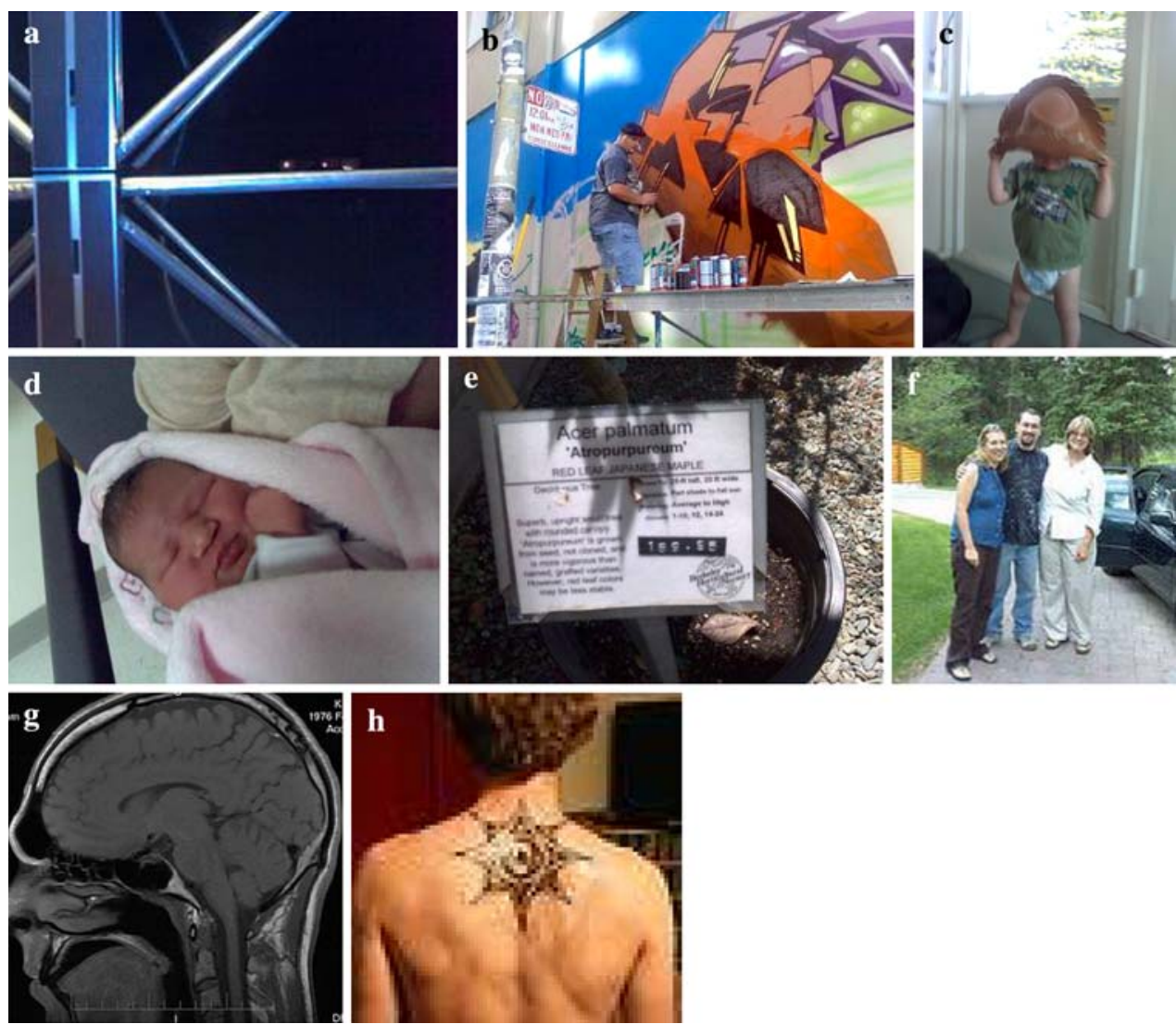
Because of this sluggishness, holding the camera still for entire period during which capture might take place is difficult and many photos were unnecessarily blurry due to hand motion (e.g. "Every photo I took was blurry.").

One participant compared the delay to digital cameras five or more years ago, saying, "Why would I want to go back?" In contrast, two participants got Apple iPhones partway through the study, and both reported that the increased speed and overall ease of picture-taking made a big difference in the amount they used their camera, despite the iPhones' lower image resolution.

\subsubsection{Image quality}

While many of the cameraphone images taken by participants were short-lived, consistent with previous research [2, 4], many of our participants wanted to archive at least a subset of the cameraphone images taken during the study. We believe that two major factors contributed to this shift. First, we had more parents than did other studies, and they took snapshots of their children, which are generally highly valued (e.g., Fig. 1c, d). Second, the improved quality of photos over previous studies resulted in more photos worth saving. The N80's camera was markedly higher quality than all but three participants' previous cameraphones, and even on par with some participants' digital cameras.

Six participants even used the N80 in place of their digital cameras. For many, the decision depended on the type of event (e.g., one said he would use the cameraphone for events "not like my son's fifth birthday, but second tier"). Others, however, said that the cameraphone images were generally good enough for them to trade the somewhat superior quality of images with their other camera for the convenience of the cameraphone and ZoneTag. Three said that they used their N80 interchangeably with their digital cameras, depending on which device was charged and at hand.

ZoneTag's instant uploading created some specific quality-related quandaries. The ability to review photos immediately is a significant benefit of digital cameras. Our participants pointed out that the typical digital photographer takes a large number of photos, and then chooses the best to share. However, with ZoneTag, the decision to upload has to be made immediately after capture, based on the image on the small screen. A major complaint was that, as on many cameraphones and even some digital cameras, the screen on the Nokia N80 was too small to assess the quality of the picture immediately after capture. More generally, immediate upload from a camera with a small display reduces the photographer's control over others' viewing experience.

The N80 camera incorporated a number of features that could improve image quality. One parent, for example, discovered that the "Sports" mode was useful for pictures of fast-moving children. However, only a few participants spent time and effort to discover these features, including some that they asked about during the interviews. Most said they did not want to bother with finding and changing settings while taking pictures and generally didn't think about it at other times. Those who did use these features were frustrated when the camera reverted to default settings, so that they would have to make their selections again.

\subsubsection{Resistances}

The reasons people do not use a new technology can be as illuminating as the reasons they do. Some dropouts did not give us a reason. One person dropped out early on because he was so dependent on his mobile phone, and his professional needs were not well supported by the study phone. However, two "refusers" were particularly interesting. One was a graphic artist for whom photography was much too important for her to be willing to adopt an "inferior" camera. After a trip she would expend considerable time and effort Photoshopping selected images and creating a high-quality website. The cameraphone could not meet her standards for image quality. The second refuser was already using a cameraphone and a passwordprotected photo-sharing site before the study; her refusal, which will be discussed below, was around the publicness of Flickr.

\subsubsection{Recommendations}

Several requirements follow from the findings above. First, clearly, the quality of cameraphone images, and the ability to adequately review them for quality, can make a significant impact on the camera's use. Recommended review features include a clear and large display, an option to quickly zoom in to view photo details, and perhaps even an automatic blur/out of focus detection and warning. Second, cameraphones are often evaluated by how they compare with "real" cameras in image quality, memory, battery life, and convenience. Image quality is not always the primary criterion; some users are willing to accept good-but-notgreat quality images in exchange for convenience. Third, users should be able to easily and comfortably capture photos at a moment's notice in a variety of environmental and social contexts with limited demands on their attention. In particular, the ability to quickly start the camera and capture a sequence of photos is a key requirement. Finally, although the adjustable camera settings were potentially useful, most users are not likely to change the automatic capture settings. Allowing settings to adjust based on the environment is desirable; and when users do manually 
adjust the settings, those should persist as long as conditions remain unchanged.

\subsection{Image uploading}

A recurring complaint about cameraphones is the difficulty people have getting images off the phone [4]. And a recurring source of procrastination among both digital and film users is the need for a separate step and a time delay getting in pictures off the camera. This is clearly an area where a technology fix would be very welcome.

A major innovation for all of our participant was the easy uploading from the phone to the Internet, as enabled by ZoneTag. After each image capture, the user is asked whether to upload the picture and, if so, is prompted to optionally enter or change metadata, including tags and a caption, or change the privacy policy. The idea of easy uploading —of being able to take and upload pictures all in one stepwas universally popular with our participants:

\section{"ZoneTag is more convenient [than using Kodak Easy Share]."}

Even heavy computer (and Flickr) users were enthusiastic, saying that the need to remember to transfer photos to their computer and then upload them to Flickr reduced their uploading. ZoneTag eliminated this later task step. People who had little free time in front of computers also liked this feature. One heavy Flickr user who didn't have broadband Internet access at home appreciated the fact that ZoneTag saved her from having to do uploading when she did have Internet access. Parents reported heavy demands on their time, and limited time online at home. For them, anything that reduced the demands on their time, especially their time at the home computer, was welcome.

Figure 2 compares image taking as recorded by the logging tool with image uploading using ZoneTag. For most of the participants, the number of uploaded images is much lower than the number of captured images. We heard several reasons for this. Some participants experienced problems with the early implementation of ZoneTag and

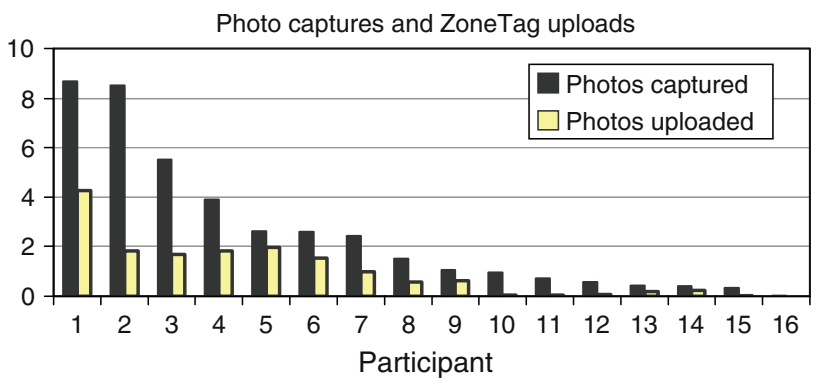

Fig. 2 Average daily numbers of captured (dark) versus uploaded (light) photos turned it off, either because of the slowness of upload discussed below, or because the application was unstable or incomprehensible.

Participants gave a range of other, perhaps more general, explanations. Some photos were basically private, and people preferred not to upload them. Many images were near duplicates, captured with the idea that only the best of a series would be uploaded.

However, once again, the specific implementation of uploading through ZoneTag was not as well-liked. These complaints are useful for understanding how people wanted to be able to upload. The biggest source of frustration was the capture-dialog delay after each capture. After each image was captured, ZoneTag asked whether to upload it to Flickr, and then displayed a screen showing settings and possible metadata (tags, location, privacy, title). Only after the user responded to this dialog (which could be engaged with, or dismissed with one click) could she or he take another photo. Photo-taking is often sequential: e.g., multiple images of the same scene (to ensure that at least one is good) or a series of images of an on-going event. In addition, picture-taking is often a part of social interaction: friends at an event, or parents taking pictures of their children. Participants did not want to be "taken out of the moment" when taking pictures (one described the ZoneTag dialog as being "bombarded with questions"), even though the upload feature was otherwise popular. ZoneTag sometimes caused them to miss the next shot, or distracted them from what they were doing as they had to turn their attention to the cameraphone. Some participants turned off ZoneTag to avoid this interruption, but if the image was not uploaded at this point, with ZoneTag it was not possible to upload it later. Some reported taking fewer pictures because of this interruption. A recurring request was for the ability to upload a batch of photos after capture.

Participants rarely found immediate uploading truly necessary. What they valued about ZoneTag was that uploading took place soon after capture, and especially that they didn't have to remember to go back and engage in extra effort to upload photos.

Another issue related to uploading was the difficulty assessing image quality on the small N80 screen, as mentioned above. If, for example, a photographer wanted to make sure they got a good image, they could neither ascertain that from the on-camera review, nor take a fast sequence of images as "insurance" and upload the best. In other words, the cameraphone-ZoneTag combination did not support established practices for ensuring successful capture. The participants who later got iPhones reported being more able to evaluate images than with the N80:

"Because the display is good, I trust it more.... With the Nokia, when I was using ZoneTag and you had to 
choose, I would upload it and then go home and see 'oh, that picture sucks.',

Due to the mostly-public nature of Flickr, participants' desire to make sure their public photos were "good" and their reluctance to go online to edit images later all make this even more important when images are uploaded immediately. The easiest workaround is to upload pictures as "private," view them online, and then change the best ones' privacy policy. However, only one participant did this. When we mentioned this solution to others, even frequent Flickr users usually saw the extra step as something they were not likely to perform.

"I do like using Zonetag. I'm too lazy to upload privately and go back later."

\subsubsection{Archiving}

This near-automatic uploading had another benefit: one parent participant described this as the feeling of having "infinite memory" on her cameraphone: she could use ZoneTag to easily delete from the phone all already uploaded photos so the memory card would never fill.

One confusing aspect of digital images generally is that they can "live" in many different places: on the camera, on one or more computer hard drives, in a Web service like Flickr, and so on. And, while some participants did back up their collections, most of them (like most personal computer users) knew they were delinquent at backing up their photos. Some participants only uploaded images to Flickr (and not to their PC), downloading selected images from Flickr to their computer. Others backed up all photos on their phones to their computer, seeing Flickr as too transitory, a service they might not continue to use, and too out of their control to be reliable backup. Many wanted to integrate their various photos collections, and wished they didn't have to do it manually. For instance, several Apple iPhoto users were interested in integrating ZoneTag and Flickr with iPhoto, since they liked the ease with which iPhoto did many operations.

A few participants discussed "backing up" digital images by getting prints. Prints were sometimes seen as easier to manage than digital photos (an older participant who used prints suggested that this practice is generational). Only one participant reported actually printing a cameraphone image during the study: a picture of a building for getting construction permits for his work.

\subsubsection{Recommendations}

Several requirements for mobile photoware arise from these findings. Mobile photoware for post-capture interaction should support a sharing-uploading-archiving option that does not interrupt sequential picture-taking or other activities in which the photographer is engaged (especially social interaction). The option to share, upload, or archive any specific image should be available at any time after the image capture. Users should also be able to select multiple images and to share or upload them as a batch.

Finally, photos could be easily or even automatically archived, integrating with users' current photo-storage mechanisms. Such archiving could enable a seemingly "infinite storage" where, since photos are backed up, they can be physically deleted from device storage to make room for more photo-taking.

Whether explicit or automatic, transferring photos over the network for sharing or archiving should be done intelligently. Possible features include background uploading (the users can continue using their devices and even their network connection, as in ZoneTag), prioritybased uploading (photos to be shared, for example, can be marked for uploading ahead of those just privately archived), and resource-aware uploading (uploading can be delayed to conserve power or cost). The status of an upload should be available and editable by the user.

\subsection{Organization and annotation}

\subsubsection{Tags and titles}

Digital images present challenges for organization and retrieval. Flickr allows tags (or keywords), titles, descriptions, and other tools for organizing and finding photos both within a collection and across photographers. A major barrier, however, is the time and effort required to enter these. Most digital cameras record date and time of capture (assuming the camera's settings are correct). Various efforts have tried to make the process of adding other metadata to photos automatic, or at least less burdensome (e.g. [26]).

ZoneTag uses its ability to sense location (via cell towers) to suggest tags used previously on the cameraphone or by ZoneTag users capturing photos in the same vicinity. ZoneTag also allowed users to set privacy and to add tags and titles to each picture at the time of capture. Figure 3 presents participants' average number of tags per photo. The tags are broken down into tags that were selected from the list of suggested tags, typed on the phone, or later added through the Flickr site. These tags do not include automatically added location tags or other tags not explicitly added by the user to their photos. Tags applied to one photo using ZoneTag persist to the following photos until the participant removes the tag or re-starts ZoneTag; these are counted as suggested tags in Fig. 3. 


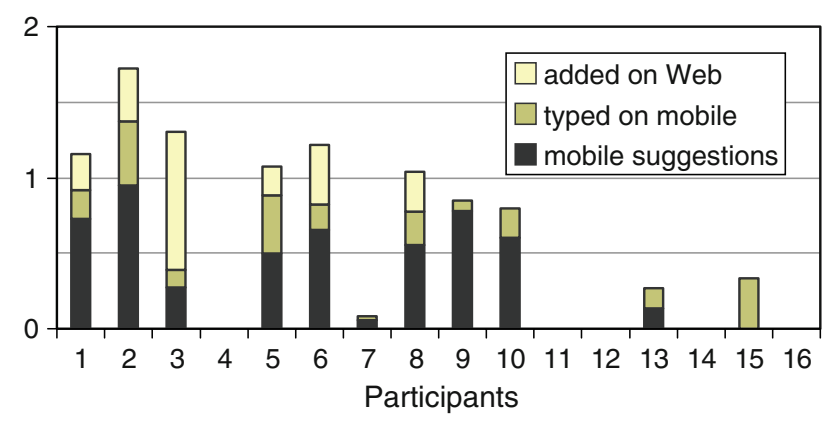

Fig. 3 Average number of added tags per photo for each participant

We noted above the some of our participants used their phones for text messaging. Not all participants had experience text messaging, but experience with other mobile text entry may reduce the perceived difficulty of tagging from the phone.

"I've done it [texting] enough. It doesn't weird me out. I do add some tags - not usually all of them."

\section{"I found myself just adding later. I am not a great texter."}

Our findings concerning annotation (including adding tags, titles or captions) through ZoneTag and Flickr echoed the findings of Ames and Naaman [19]: when participants annotate their photographs, they consider multiple audiences (friends and family, the general Flickr audience, and themselves). They want to make their photographs organized or searchable, and add contextual information and explanation to the photo.

Participants with prior Flickr experience were most comfortable with tagging and most familiar with its benefits. Some viewed tagging as something they were "supposed to do" as a contribution to the larger Flickr community. Participants who were not as familiar with tagging or Flickr often felt that tagging wasn't worth the effort. Several parents said that every picture would have the same tags (e.g., their children's names) anyway. Many participants tended to add titles to their images instead, since titles were much more visible to novice Flickr users and were more familiar to participants who had used other photo-sharing systems. Some participants commented that images with titles were simply more interesting.

Overall, date of capture and user-added titles were cited as the most useful metadata. As other research has found concerning both cameraphone images and other digital and paper images [4], our participants generally used time as a primary organizing principle for their own photographs, both on Flickr and on the desktop.

ZoneTag used location information to suggest tags previously used in the same area by this and other ZoneTag users. This feature was considered as convenient by some:
"I like ZoneTagging. [It] offers tags for stuff close by, and that's really convenient."

For other participants the suggestions created confusion. Even slight inaccuracies-suggesting a tag for the next neighborhood over, or showing seemingly unrelated tags that happened to have been used by others in the same location, such as the names of strangers-annoyed users. Some participants' expectations were frustrated when ZoneTag performed unevenly, at times suggesting the exact restaurant they were in (or, in one fortuitous case, the name of a friend who had not previously been tagged), and at other times not suggesting anything useful. Overall, most participants, even technologically savvy ones, found the tag suggestions opaque: they didn't want to have to figure out how ZoneTag made suggestionsthey just wanted it to work, predictably and reliably, as also shown by Naaman and Nair [26].

\subsubsection{Location metadata}

Location-aware services are a major area of development for mobile technologies. Location is often an important element of a photo, since sights and activities are often associated with a specific place. On Flickr, images of the same place taken by various photographers can be retrieved. One of the major features of ZoneTag is that it automatically adds location information, including, when available, name of town, state, and zip code, and sometimes neighborhood and even the name of a café, theater, or the like, if these have been entered by a user previously or are available in the Yahoo! database. Since the N80 does not have built-in GPS, ZoneTag approximates the photo capture location using cell tower data, when available.

Most participants were only interested in location information on their own photos when they were in new or unusual places, such as on vacation. Unfortunately, since the cell tower data availability was sparse outside the immediate geographical region of the experiment, many vacation spots were not covered; the location features of ZoneTag then depended on the participant manually entering the location, which very few did. However, once one ZoneTag user associated a specific cell tower with a location, any ZoneTag user had access to that location information.

Several participants who worked for a technology company and described themselves as "metadata-oriented" were strongly interested in having location data for their photos. They wanted to have the option to use any possible metadata for their photos, and argued that, since the service provider had location information for their phones, they wanted access to it, too. Some said that having those tags on their own photos, though not particularly useful now, 
could be useful in the future-a "nice plus," and "just another way to navigate."

Location information can raise privacy concerns. A few participants were mildly concerned about privacy, especially when location data was attached to their house or places their children spent time. However, the location was vague enough (i.e., 5-digit postal code level) that no one saw it as a real threat.

While most participants didn't care much about location information on their own photos, they did like it on others' photos. At least one person used Flickr photos to research potential vacation sites. Four participants enjoyed using the location data to surf on their phones using Zurfer, or from their laptop, for other Flickr photos taken in their neighborhoods or their current location. One participant who attended a concert and found, from the suggested tags, that the theater had already been "ZoneTagged" went to Flickr later to look at the photos uploaded by people who had tagged that location, reasoning that if they had this one interest in common, they might have others.

\subsubsection{Recommendations}

Though some users are not interested in annotating their photos, designing photoware to support and motivate annotation can create long-term value for users, viewers, and service providers. Annotation should be possible at any time, even after the photo is uploaded, and perhaps made into a "downtime" activity. (As we'll discuss below, some participants viewed images on the phone while commuting, and said they could use that time to annotate images.) Aiding users with relevant suggestions for annotation could be beneficial, but these suggestions should be transparent (i.e., users should be able to understand why a certain suggestion is made) and consistent.

In terms of automatic metadata, date, time, and location metadata are most interesting to users. Location metadata should be consistently available and correct, or at least communicate a confidence level and give an explanation when location data is missing. Photos could be automatically associated with location data, as long as the user can control both the exposure and the location level of granularity (e.g., "only friends can see it and never expose the exact coordinates"), as well as modify this preference for individual of sets of images (e.g., vacations).

\subsection{Sharing}

A major use of photos of all kinds is sharing: with copresent others, and across space and time. The family photo album, for example, is designed to collect, archive, and share photos. All participants reported at least some motivation to share photographs with friends or family, and parents in particular were under pressure from extended families to keep a steady stream of images coming.

Frohlich et al. [6] discuss image-sharing along two dimensions: time (synchronous vs. asynchronous sharing) and location (copresent vs. distant sharing). We propose two additional dimensions. First, another aspect of the time dimension that affects sharing is how quickly the picture is shared after being taken, which is increasingly relevant with cameraphones. Second, online sharing, such as that through Flickr, adds an audience dimension where photos can be shared with a set of known others or with the general public.

\subsubsection{Sharing Face-to-Face}

Consistent with previous findings on face-to-face photo sharing $[6,8]$, showing images to others on the phone's screen was popular with almost all participants. Face-toface sharing allows the owner to customize the selection of images and the stories told to the viewer. Figure 4 shows how often users view images using the Gallery function on the phone, either to show images to others or to view images themselves. Several discovered and used the phone's "slideshow" feature for displaying photos. Some of our participants saw this kind of sharing as the trend for the future. As one explained, "inviting [people] over, pouring some tea, and seeing pictures just isn't something that people do anymore." The phone provided a more convenient, informal mechanism for sharing photographs. Naaman et al. [24] report that co-present sharing on the phone screen was a common task for many Zurfer users. Much of that use was geared towards photos taken by the user, for story telling, illustration, or "identity presentation" purposes.

\subsubsection{Sharing at a distance}

We recruited social groups in the expectation that they would share with one another. However, most photo sharing was with distant family and friends, a reminder that

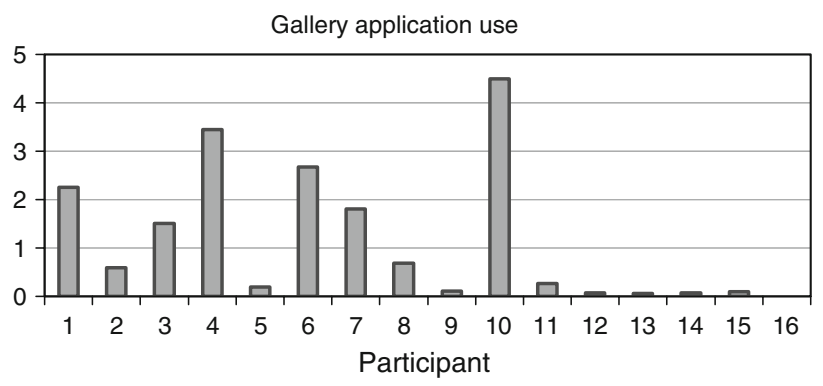

Fig. 4 Frequency of photo viewing on the phone 
social networks are complex and varied and so are the uses of photos.

Some participants were prior users of Flickr. These were generally the most comfortable with and enthusiastic about using Flickr. They were in the habit of posting their photos and viewing their friends' photos online. Several reported using relying on Flickr to keep their friends and family upto-date-and, in turn, keeping up with their friends. These tended to be the most enthusiastic about ZoneTag because it made something they were already doing, uploading images to Flickr, faster and easier.

This sharing via Flickr-and, less often, other mechanisms - took two forms. The first was to maintain what Van House [18] calls "distant closeness," especially through photographs that document experiences or say "I'm thinking of you" (consistent with Kindberg et al. [25]). The other kind of sharing involved delivering more functional, often time-sensitive, information such as shopping choices or work photographs, which was also found in earlier cameraphone studies [2, 4]. One participant used ZoneTag to share photos (via Flickr) with a coworker in Europe to facilitate decision-making, augmenting them with a phone call. One participant who was undergoing medical procedures felt uncomfortable talking about them, so he posted annotated pictures to Flickr to explain his condition to friends and family without having to talk about it.

Several Flickr users preferred "passive" sharing on Flickr to more directed sharing such as through email because they didn't have to worry as much about sharing too many photos - their viewers could choose which photos to see and wouldn't end up with an over-full inbox.

Participants who hadn't used Flickr before the study, for the most part, tended to continue to share in ways consistent with prior practices and established communication channels such as email or other online sharing services. Several said that they didn't want to make their distant friends and family sign onto yet another online service.

In sum, when possible, participants folded their cameraphone photos into their existing image-sharing practices. They were sensitive to the recipients' technological sophistication and communication habits as well as to what the receiver would want to see ("I don't want to burden them").

\subsubsection{Privacy and audience}

Privacy is a major concern with online posting of potentially sensitive information. Ahern et al. [13] note that photographs are particularly revealing, often exposing personal and contextual information. The authors also note, however, that privacy decisions are complex, embracing security, social disclosure, identity and convenience. While many participants said that privacy was, in theory, a concern, most had never suffered any harm from having their data public and didn't see any actual threat.

We found that most participants adopted blanket strategies for photo privacy (many of them always or nearly always uploading photos as public). Nevertheless, participants expressed a recurring desire to be able to fine-tune access to images according to a more refined differentiation of audience than Flickr allows, but with little or no added effort. In other words, most weren't concerned about security, but many were concerned about social disclosure. They wanted to be able to vary the privacy controls by image and by recipient to a much finer degree than is currently possible on Flickr.

We noted above that one "refuser" chose not to use ZoneTag and Flickr primarily for privacy reasons. She was already using a cameraphone and a password-protected sharing site. Although Flickr's privacy settings are flexible, she had no interest in a site that allowed public sharing and was happy with the site she was using. In addition, she was uncomfortable with viewing others' images, even public images. For her, a public photo sharing site did not fit her concept of appropriate use of photos.

Participants repeatedly said that they were not likely to log onto Flickr later to upload or edit photos. They were also averse to having to go online to manage privacy settings. They wanted near-automatic yet finelytuned privacy management at time of upload from the phone. Ironically, the same barriers that some complained about as limiting their sharing with less computer-savvy others were sometimes seen as convenient filters:

"I've sent a few links to my parents. [T]hey really enjoyed seeing the photos of my cross country trip. And it's probably beneficial to me that they don't understand how to navigate everything so they don't see my photos from Burning Man. [...] They absolutely don't understand how to do that."

\subsubsection{Recommendations}

Image-sharing - at a distance, and with co-present othersis an important motivator in photography. Overall, mobile photoware should integrate easily with the various archiving and sharing methods and services with which users (and photo recipients) are already engaged. Face-to-face sharing remains popular, but casual and "one-on-one"; convenient access to the full-screen slideshow feature (and perhaps even customizable slideshows) helps support this kind of viewing.

Recommendations for sharing and privacy for mobile photoware include an addressee-driven privacy and sharing model in addition to policies based on enduring categories 
such as "family only." Users should have maximum flexibility in defining groups and viewing permissions, which should be customizable for individual photos or batches.

\subsection{Mobile viewing}

Many of our participants regularly looked back through at least some of their photographs on their mobile phones by themselves, in addition to sharing them face-to-face. Figure 4 shows how frequently each participant viewed images (by themselves or showing them to others) using the Gallery application on the phone.

Participants told us that they sometimes started the Gallery application on the cameraphone (the native imageviewing application) looking for a specific photo (perhaps to share or archive) and stayed to browse. Other times, participants used photo-viewing as a distraction while bored or as a type of "play" in place of other downtime activities such as reading the paper. For instance, one participant reported using Zurfer extensively in lines, during his commute, and in the bathroom. Some people commented that they wished they could also upload and add annotations while browsing in this way, as it was when they would be most likely to have the time and interest to do so. Zurfer was also used to make sure photos had uploaded properly without having to wait for computer access.

Beyond their own content, we have noted that some of our participants also used the social features of Flickr and Zurfer to keep up with friends through images. For instance, those with friends on Flickr would visit the site up to several times a day or use Flickr's RSS feature to monitor friends' photo-streams. (They could also have used Zurfer, but few tried out Zurfer long enough to realize this.) Social usage was the major driver of Zurfer use in the Naaman et al. [24] study.

The Zurfer feature that allows viewing "nearby images" at the user's current location was also used as a source of entertainment. Several participants who didn't have contacts in Flickr still enjoyed using Zurfer to look at photos taken in certain locations. These locations included not only their current location but also "favorite" places like their neighborhood, their hometown, or the location of a friend.

\subsubsection{Recommendations}

Many people find viewing photographs enjoyable; many of our participants used it as a downtime activity such as while on public transit. People view friends' images as ways of keeping up on one another. Users should have easy access to their own photographs, ideally their entire collection and not just photos captured recently. In addition, access to friends' photos and other interesting collections, such as those based on location, could be driving both consumption and capture use. Many phones come with games, but more personal and meaningful downtime applications, like viewing, annotating, and sharing photos, would likely be more fulfilling for some. An environment where users can post photos and get immediate responses could be welcome.

\subsection{Technical considerations}

Some technical problems, particularly the lag of the camera and ZoneTag, the small screen, and blurry images, were discussed above. In addition, the Nokia N80 was generally slow to respond. Moreover, when using ZoneTag, its battery barely lasted through a day, even with most other battery-intensive services, such as WLAN search, disabled. Participants found such poor performance unacceptable. A mobile device meant for frequent and varied use should respond quickly and be more intelligent about maximizing battery life.

Both ZoneTag and Zurfer were prototypes, and both crashed on occasion. The initial implementation of the monitoring software tended to fill up the phones' memories. We replaced this early on, but not before some participants were frustrated by the phones crashing. Participants reported that even small problems curtailed their use, especially early in their experience. Overall, it is important that even experimental applications are reliable.

Two of our participants bought Apple iPhones during the study and were able to compare them to the Nokia N80 in the final interview. Though their iPhones were also prone to crashing and the camera was lower-resolution than the Nokia N80's, the image display was large and clear, the battery lasted longer and the phone automatically synched with iPhoto, when connected to their computer. Both of these participants reported taking more images with their iPhones and sharing them with more people. While some of this may be novelty (each had had the iPhone for about a month when we discussed it with them), some is probably also due to the relative ease of use.

ZoneTag requires an Internet data plan in addition to a voice plan, which allows for Web access beyond just ZoneTag. Many participants started checking their email or doing Web searches from the phone during the study. Several participants said that they had become "addicted" enough that they were going to keep their data plan after the study. Most who were using the data plan only for ZoneTag didn't think the added cost $\mathbf{( \$ 2 0 / m o n t h )}$ was worth the convenience. For mobile photo uploading and browsing to really take off, costs will have to be much less or even nonexistent (or perhaps bundled with the standard 
phone plan or other applications). Otherwise, phone applications should be marketed with other data service uses like email and Internet browsing to justify the cost and maximize the benefits from the data plan. Once again, we see the value of an assemblage of hardware, software, and services: the total set of interrelated components made the entire bundle more desirable.

\section{Requirements for mobile photoware}

While specific requirements are listed in the various sections above, here we repeat and review a few major themes in requirements for mobile photoware. It is our contention that mobile photoware will be increasingly prevalent, replacing many "ordinary" digital cameras, at least for many purposes, in the near future. So our findings from this study may be useful for these future design developments.

First and foremost, we believe that mobile photoware should take a holistic approach for device-based media, including integrated (but flexible) support for capture, archiving, sharing, annotation, viewing and reviewing. Ideally, the device should be able to tie into a variety of existing technical ecologies (online sharing sites, desktop image browsers, etc.) and user practices. Most people already have large collections of images, into which they want to integrate new images. Many already have hardware and software solutions to part of the image lifecycle, such as iPhoto, that they already use and are satisfied with. And images are frequently shared with others, through established means such as email and, increasingly, through social network services like Facebook. New technologies need to be compatible with existing and emerging practices and preferences. Many of our participants were sharing with friends and family who varied considerably in their current uses of and comfort with technology; yet, overall, Internet users are increasingly comfortable with a variety of image-related activities. Our approach integrating cameraphone, ZoneTag, and Flickr (and, to a lesser extent Zurfer) was generally wellreceived; but activities requiring extra effort (such as going online to change a photo's privacy policy) were major barriers, for some users, to sharing but even to picture-taking.

The mobile device should allow for viewing, reviewing and sharing the user's own images, ideally from all sources, not limited to photos taken with the specific device. "Photo wallet" and "slideshow" features are key in this context. Ideally, other users' public photos should be viewable on the device. Such mobile viewing, to be used in various mobile downtime situations (waiting in line, on the bus) could be also leveraged for performing annotation and other image-related tasks while mobile.
Ease and convenience of use are, naturally, key requirements. In some ways, this is straightforward. Mobile photoware should minimize interruption and disruption in the user's established photo-taking flow, allowing maximum flexibility for the user to review, annotate, share, and perform other actions on these media at any convenient time.

Image annotation is a useful feature, but most users do not want to bother themselves with it on constant basis, especially from the phone's limited keyboard. Simply marking "important" photos could be a lightweight way to put order into the capture chaos. Batch annotation, perhaps tied to sharing activities, and done during "downtime," is likely to be more useful. Automatic location metadata is often useful, especially in such scenarios as travel. Other system-suggested annotation and metadata has to be carefully designed to be relevant and transparent.

The "infinite memory" nature of the network-connected cameraphone is a major benefit that should be preserved, expanded, and capitalized on. Two major constraints on digital photography are battery power and memory. But some of our users saw the connectedness of the cameraphone as freeing them from the second of these, creating a sense of no limits to photo taking, and unlimited availability of photos on the device. This requires, however, that people be able to trust the camera and the network, to know that their photos are safely uploaded and stored. More generally, we argue that mobile technologies that are effective as portals to the vast resources of the Internet are more likely to be successful. While not many of our participants used their cameraphones for email and Internet searching, since then the iPhone has increased people's expectations of the mobile device as Internet-enabled. For some of our participants, the overall combination of services was the so-called "killer app:" the cameraphone as a device for image capture and uploading, for viewing images via Zurfer, for email and internet searching - the overall package was much more alluring than any one application.

Sharing is one of the major drivers of photography in general and cameraphones in particular. Instant sharing, we discovered, is not as important as easy and timely uploading and sharing. Successful and satisfactory sharing is also dependent on the sharer's ability to verify that what gets shared is what's intended. Uploading from the phone is complicated by the difficulty of assessing image quality on the spot. If the image fails to capture the intended sight, or is of unsatisfactory clarity or technically quality, the sharing is a failure. The image view on the phone, therefore, needs to be of high enough quality to reassure the sender that the image is a success.

Image sharing is a social interaction. Sharing mechanisms need to be flexible but not burdensome, suiting the 
abilities, preferences, and technologies of all parties to the transaction.

Here it's useful to consider our "refuser" whose primary complaint was the quality of the images and their presentation. While it may be a while before cameraphones and online sharing sites like Flickr can satisfy this kind of "serious" artist, since our study, Flickr has added some elementary photo adjustment software for functions like cropping and exposure compensation. And the quality of cameraphone images and functions is continually improving. While some users prefer not to have to investigate cameraphone adjustments or to manipulate images after capture, this kind of user is likely to appreciate just such functionality. The increasing use of images for purposes like Facebook may also raise expectations about image quality, making at least some users more interested in this added functionality.

The main privacy concern around photos that we heard about is social disclosure: what does the photographer want whom to know about what? Allowing photo privacy policies determined by a small set of enduring categories (such as "friends only") is useful, but users should be able to share media with specific individuals through fine-grained modifications of a photo's privacy policy. Sharing modes can be single image as well as batch or a set of photos from a single event; and images could be shared with a set of specific individuals.

\section{Other implications}

From this study we can also draw some inferences about mobile devices, including mobile imaging devices, more generally. Usability is of course always important, but participants used "easy" and "lazy" in more complex and varied ways. Some people who frequently logged onto Flickr to view friends' images still described themselves as "too lazy" to go online and tag or change settings on their own images. Mobile devices are used across and during variable situations, so at one time completing a task (e.g., annotation) may interrupt on-going activity while at another time it may be welcomed and be labeled "easy".

New technologies have to fit people's on-going practices related to those technologies and the situations in which they will be used. People are used to being able to snap a series of photos without interruption; the ZoneTag dialog broke their usual chain of activity. Expectations for new technologies are based on people's experiences with what they see as related technologies. In this case, the cameraphones were seen as "sluggish", but also possessing "infinite memory", compared specifically to digital cameras.

With cameraphones, Zonetag, and Flickr, we also saw complex issues of the changing temporality of photography.
The delays in uploading and sharing inherent in other kinds of cameras were alleviated. For most purposes, "immediate" uploading was unnecessary, but "relatively soon" was highly valued. In fact, rapid sharing can create new problems, as viewers might see an image on their computer screen that the photographer had only seen on the inadequate camera screen.

Among our participants, the users most satisfied with the new technology were those for whom it was an incremental change in prior technologies and practices, and for whom it solved a problem: prior cameraphone and Flickr users who liked automatic uploading; prior Flickr users who liked the ubiquity of the cameraphone and the convenience of ZoneTag; prior cameraphone users who like the addition of ZoneTag and Flickr; and so on. Those least satisfied were those for whom these technologies either were far from what they were already doing, or a poor substitute for practices established with recipients. This is a useful insight for other studies trying to decide how to test new technologies.

\section{Conclusions}

In this study, we saw how "ordinary" users incorporated networked cameraphones into their daily lives. They used the devices to capture and upload images, but also for showing and viewing images on the phone. The significance of the portability of the cameraphone extends beyond capture to the many aspects of mobile photoware.

The potential of cameraphones as ubiquitous, high quality image-capture devices, combined with cheap online storage and easy sharing, could change the face of consumer imaging. The always-at-hand, "infinite memory" possibilities of networked cameraphones may well change personal photographic practices. One of the strengths of this study was that we provided a complete assemblage of hardware and software to support the full life-cycle of images. We found that all parts of this assemblage have to work easily, and well, both with each other and with other image-related technologies. For example, automatic uploading places increased the significance and demands of on-screen viewing; the default public nature of Flickr complicates the management of privacy and social disclosure; and iPhoto desktop users wanted the entire assemblage to integrate well with iPhoto and their other images.

While some of our lessons offer little surprise, they are strongly grounded in a long-term empirical study. Overall, we saw many diverse patterns of use. While we noted some barriers and sources of dissatisfaction, most participants used and were enthusiastic about this assemblage of technology and services. They overwhelmingly liked automatic uploading from the phone to Flickr. Some liked the 
automatic location tagging, especially when traveling. Many used their mobile devices to view images and to share them with others.

This focus on specific technology has been of necessity a limited study. In the future we hope to expand our consideration to other media, including video, and also different cultures and demographics. In this kind of research, it's inappropriate to generalize much beyond the highlysituated experiences of our specific user community and their circumstances. However, our findings, grounded in a comprehensive study of "ordinary" users, should be useful for designers of future cameraphone-based systems.

Acknowledgments We would like to thank Nathan Good and Vlad Kaplun; the participants in our study; and the anonymous reviewers.

Open Access This article is distributed under the terms of the Creative Commons Attribution Noncommercial License which permits any noncommercial use, distribution, and reproduction in any medium, provided the original author(s) and source are credited.

\section{References}

1. Okabe D, Ito M (2006) Everyday contexts of camera phone use: steps toward technosocial ethnographic frameworks. In: Höflich JR, Hartmann M (eds) Mobile communication in everyday life: ethnographic views, observations, and reflections. Frank \& Timme, Berlin

2. Kindberg T, Spasojevic M, Fleck R, Sellen A (2005) The ubiquitous camera: an in-depth study of camera phone use. Pervasive Comput 4:42-50

3. Koskinen I, Kurvinen E, Lehtonen T-K (2002) Mobile Image. IT Press, Helsinki

4. Van House NA, Davis M, Ames M, Finn M, Viswanathan V (2005) The uses of personal networked digital imaging: an empirical study of cameraphone photos and sharing. CHI ' 05 ext abs, Portland, OR, USA. ACM Press, New York, pp 1853-1856

5. Van House NA (2007) Flickr and public image-sharing: distant closeness and photo exhibition. CHI '07ext abst San Jose, CA, USA. ACM Press, New York, pp 2717-2722

6. Frohlich D, Kuchinsky A, Pering C, Don A, Ariss S (2002) Requirements for photoware. In: Proceedings of CSCW 2002, New Orleans, Louisiana. ACM Press, New York, pp 166-175

7. Chalfen R (1987) Snapshot versions of life. Bowling Green State University Popular Press, Bowling Green

8. Crabtree A, Rodden T, Mariani J (2004) Collaborating around collections: informing the continued development of photoware.
CSCW '04, Chicago, Illinois, USA. ACM, New York, pp 396405

9. Rose G (2004) 'Everyone's cuddled up and it just looks really nice': the emotional geography of some mums and their family photos. Soc Cult Geogr 5:549-564

10. Rodden K, Wood KR (2003) How do people manage their digital photographs?. In: Proceedings of CHI 2003, Ft. Lauderdale. ACM Press, New York, pp 409-416

11. Kirk D, Sellen A, Rother C, Wood K (2006) Understanding photowork. In: Proceedings of CHI '06, Montreal. ACM, New York, pp 761-770

12. Miller AD, Edwards WK (2007) Give and take: a study of consumer photo-sharing culture and practice. In: Proceedings of CHI '07, San Jose. ACM Press, New York, pp 347-356

13. Ahern S, Eckles D, Good N, King S, Naaman M, Nair R (2007) Over-exposed?: privacy patterns and considerations in online and mobile photo sharing. In: Proceedings of CHI '07, San Jose, California. ACM Press, New York, pp 357-366

14. Counts S, Fellheimer E (2004) Supporting social presence through lightweight photo sharing on and off the desktop. In: Proceedings of CHI '04, Vienna. ACM Press, New York, pp 599606

15. Davis M (1993) Media streams: an iconic visual language for video annotation. Telektronikk 4.93:59-71

16. Salovaara A, Jacucci G, Oulasvirta A, Saari T, Kanerva P, Kurvinen E et al. (2006) Collective creation and sense-making of mobile media. In: Proceedings of CHI '06, Montreal. ACM Press, New York, pp 1211-1220

17. Sarvas R, Viikari M, Pesonen J, Nevanlinna H (2004) Mobshare: controlled and immediate sharing of mobile images. Multimedia 2004, New York. ACM Press, New York

18. Van House NA (2006) Distant closeness: cameraphones and public image sharing. UBICOMP '06 PICS Workshop

19. Ames M, Naaman M (2007) Why we tag: motivations for annotation in mobile and online media. In: Proceedings of CHI '07, San Jose. ACM Press, New York, pp 971-980

20. Van House NA (2006) Interview viz: visualization-assisted photo elicitation. CHI '06 ext abs, Montreal. ACM Press, New York, pp 1463-1468

21. Flickr http://flickr.com

22. ZoneTag http://zonetag.research.yahoo.com/

23. Zurfer http://zurfer.research.yahoo.com

24. Naaman M, Nair R, KaplunV (2008) Photos on the go: a mobile application case study. In: Proceedings of $\mathrm{CHI}$ '08, Florence. ACM Press, New York, pp 1739-1748

25. Kindberg T, Spasojevic M, Fleck R, Sellen A (2005) I saw this and thought of you: some social uses of camera phones. Ext Abs CHI '05, Portland. ACM Press, New York, pp 1545-1548

26. Naaman M, Nair R (2008) ZoneTag's collaborative tag suggestions: what is this person doing in my phone? IEEE Multimed 15(3):34-40 